\title{
One Year of COVID-19: The "New Normal"
}

\author{
Prasenjit Mitra $^{1} \cdot$ Sanjeev Misra ${ }^{2} \cdot$ Praveen Sharma $^{1}$
}

Published online: 5 January 2021

(C) Association of Clinical Biochemists of India 2021

The entire world was unaware of the term COVID-19 or SARS-CoV-2 a year ago. It all began in December 2019, when the first case presented with pneumonia of unknown origin in the small Chinese town of Wuhan. The country office of WHO, China, was informed about the same on December 31, 2019. The rapid global spread along with high morbidity and mortality led to the declaration of an International Public Health Emergency by January 2020. The causative agent of this disease was a novel member of the coronavirus family and thus was initially named novel Coronavirus (nCoV) 2019. Members of the coronavirus family, the SARS-CoV and MERS-CoV have been responsible for SARS and MERS respectively in 2003 and 2012. By February 2020, the International virus classification commission named this novel entity the severe acute respiratory syndrome coronavirus 2 (SARS-CoV-2), and WHO declared the name of the disease as Coronavirus disease 2019 (COVID-19). The unstoppable spread and public health burden that COVID-19 ushered in the entire world compelled WHO to declare it a pandemic on March 11,2020 , an announcement which several agencies considered to be quite late [1]. As on 24th December 2020, the total number of cases and deaths stand to 79,132,197 and $1,739,149$ respectively. Presently, there are 21,674,999 active infected patients, among which 21,568,517 (99.5\%) are in mild condition while remaining 106,482 (0.5\%) are in serious or critical condition. During the first three

Praveen Sharma

praveensharma55@gmail.com

1 Department of Biochemistry, All India Institute of Medical Sciences, Jodhpur, India

2 Department of Surgical Oncology, All India Institute of Medical Sciences, Jodhpur, India months, the most affected countries included the United States of America (USA), Italy, Spain, China, Germany, and Iran. In India, the cases of COVID-19 started increasing rapidly by the end of March 2020 to the extent that a nationwide lockdown was implemented to break the chain of spread. Presently, India ranks 2nd with 9,703,908 COVID-19 cases and is following the United States of America (USA) with 15,370,339 total cases [2].

Earlier reports described COVID-19 as a severe respiratory illness. Individuals suffering from COVID-19 may experience different symptoms which could be implicated to differences in the type of infected cells and tissues, variation in the Angiotensin-converting enzyme 2 (ACE2) receptors expression, as well as, varied immune response to the virus by an individual. Furthermore, comorbidities like old age, hypertension, diabetes mellitus (DM), coronary heart diseases, obesity, and cerebrovascular disease are one of the most important determining factors leading to different disease outcomes [3].

Several studies have implicated hyperactive immune response and cytokine storm to be the preliminary cause of the disease severity in infected individuals. $88 \%$ similarity in the genomic structure shared by SARS-CoV and SARS$\mathrm{CoV}-2$, has enabled the scientists to explain the similar mode of viral entry and immune evasion strategy followed by the virus. The SARS-CoV-2 virus dictates its entry into the host by binding to ACE2 receptors. Therefore, the main target cells include those that express ACE2 receptor, i.e. the bronchial epithelial cells, alveolar epithelial cells, vascular endothelial cells and macrophages in the lung and pneumocytes of alveolar epithelium. On binding to the receptor, there is aggressive inflammatory response, autophagy, basal membrane detachment and inhibition of ACE2 expression, which in turn, result in in acute lung injury, by inducing angiotensin II binding to the 
Angiotensin II receptor type 1 (AT1aR) receptor. Cytokine storm resulting in a surge of inflammatory cytokines, chemokines and free readicles leads to severe damage to lungs and other organs thus causing multi organ failure and finally death in severe cases [4].

A lot of studies have also proposed the genetic makeup of the host to play an important role in disease susceptibility and severity. Genetic variants of ACE2, transmembrane protease serine 2 (TMPRSS2) and dipeptidyl peptidase-4 (DPP4) expression, as well as, human leukocyte antigen (HLA) and major histocompatibility complex (MHC) gene could be a potent risk factor for disease suspectibility, as well as, severity. Although, innumerable clinical trials for COVID-19 management have been on going worldwide, unravelling such high risk variants would open up new potential therapeutic targets for leading effective treatment [5].

The onset of COVID-19 pandemic has provided humanity with new challenges, in terms of health care infrastructure development, as well as, improvement, capacity building, research, teaching, and mental health. The researchers have been busy learning the details of the virus, the disease characteristics, the risk factors, the therapeutic options and the possibility of development of a vaccine. Due to the unavailability of a specific cure against the SARS-CoV-2, preventive measures like appropriate hand hygiene, social distancing and wearing protective masks have been promoted globally. The world has gone through a lot in this past year, and many hypotheses were modified based on findings from well organised multicentric clinical trials. Now that the emergence of a possible vaccine is on the horizon, we may hope that the situation gets better with this new year and we win over this pandemic. If not, then the only possibility is to live with the "new normal", until an effective cure is established.

\section{References}

1. Mitra P, Misra S, Sharma P. COVID-19 Pandemic in India: What lies ahead. Ind J Clin Biochem. 2020;35:257-9. https://doi.org/10. 1007/s12291-020-00886-6.

2. WHO Coronavirus Disease (COVID-19) Dashboard n.d. https:// covid19. who.int. Accessed 24 Dec 2020.

3. Mitra P, Suri S, Goyal T, Misra R, Singh K, Garg MK, et al. Association of comorbidities with coronavirus disease 2019: a review. Ann Natl Acad Med Sci (India). 2020;56:102-11. https:// doi.org/10.1055/s-0040-1714159.

4. Lingeswaran M, Goyal T, Ghosh R, Suri S, Mitra P, Misra S, et al. Inflammation, immunity and immunogenetics in COVID-19: a narrative review. Ind J Clin Biochem. 2020;35:260-73. https://doi. org/10.1007/s12291-020-00897-3.

5. Choudhary S, Sreenivasulu K, Mitra P, Misra S, Sharma P. Role of genetic variants and gene expression in the susceptibility and severity of COVID-19. Ann Lab Med. 2021;41:129-38. https://doi. org/10.3343/alm.2021.41.2.129.

Publisher's Note Springer Nature remains neutral with regard to jurisdictional claims in published maps and institutional affiliations. 